\title{
Molecular Diagnosis of Sexually Transmitted Infections: A Diverse and Dynamic Landscape
}

\section{CHRIS L. MCGOWIN, RODNEY E. ROHDE, GERALD REDWINE}

\begin{abstract}
ABBREVIATIONS: CDC - Centers for Disease Control and Prevention, CT/NG - C. trachomatis and N. gonorrhoeae, FDA - Food and Drug Administration, HPV - Human Papilloma Virus, LDT - laboratory-developed test; MDx - molecular diagnostics, NAAT - nucleic acid amplification test, NGU - non-gonococcal urethritis, PCR - polymerase chain reaction, PID - pelvic inflammatory disease, POC - point of care, STD - sexually transmitted disease, STI - sexually transmitted infection, TMA - transcription mediated amplification
\end{abstract}

INDEX TERMS: Molecular diagnostics, diagnostics, sexually transmitted disease, sexually transmitted infection, Trichomonas vaginalis, trichomoniasis, Human Papilloma Virus, HPV, cervical cancer, Mycoplasma genitalium, non-gonococcal urethritis, NGU, cervicitis

\section{Clin Lab Sci 2014;27(1):40}

Chris L. McGowin, PhD, Louisiana State University Health Sciences Center, Department of Microbiology, Immunology and Parasitology, New Orleans, LA

Rodney E. Rohde, PhD, MS, SV, SM(ASCP) ${ }^{C M} M B^{C M}$, Clinical Laboratory Science Program, College of Health Professions, Texas State University, San Marcos, TX

Gerald Redwine MEd, MT(ASCP), Clinical Laboratory Science Program, College of Health Professions, Texas State University, San Marcos, TX

Address for Correspondence: Chris L. McGowin, PhD, Louisiana State University Health Sciences Center, Department of Microbiology, Immunology and Parasitology, 1901 Perdido St.; MEB 6214, New Orleans, LA 70112-2822, 504 568-7281, cmcgow@lsubsc.edu

More than 300 million curable sexually transmitted infections (STIs) are acquired each year worldwide ${ }^{1}$ making these infections a significant public health and economic concern. At least 25 urogenital and reproductive tract diseases have infectious etiologies that are transmitted through sexual contact and the CDC conservatively estimates approximately 110 million STIs exist among American men and women. ${ }^{2}$ The roughly 20 million incident cases in the USA alone have direct costs for treatment that top more than $\$ 15$ billion. ${ }^{2,3}$ Sexually transmitted pathogens are extraordinarily adapted to life in the urogenital tract. This is exemplified by the fact that all STIs can persist longterm in the face of extensive and complex host innate and adaptive immune responses. This is particularly true in females who disproportionately bear the prevalence and pathological sequelae of STIs with inflammatory syndromes like pelvic inflammatory disease (PID), tubal infertility and several pregnancyrelated complications such as preterm birth and spontaneous abortion. Polymicrobial syndromes like bacterial vaginosis and some cases of PID are now recognized as significant medical concerns and have also contributed directly to our understanding of the microbiome and its impact on reproductive health. This FOCUS series of articles has been dedicated to three organisms that creatively illustrate the spectrum and importance of molecular diagnostics (MDx) for STIs: Mycoplasma genitalium, Human Papilloma Virus (HPV), and Trichomonas vaginalis.

The last twenty years have been dynamic and exciting for the field of molecular diagnostics. Since the introduction of the first FDA-approved nucleic acid amplification test (NAAT) for $C$. trachomatis and $N$. gonorrhoeae (CT/NG) in the early 1990's, the market and technologies have burgeoned and have been led largely by diagnostic tests for STIs. Currently, more than 25 FDA-approved NAATs exist for CT/NG alone, and the menu is rapidly expanding to include new STIs like $M$. genitalium and pathogens of renewed importance like $T$. vaginalis. Collectively, the field of STI diagnostics is enormous, both in the diverse array 


\section{FOCUS: MOLECULAR DIAGNOSIS OF SEXUALLY TRANSMITTED INFECTIONS}

of testing platforms and in the magnitude of tests performed worldwide. As such, the market for STI diagnostics is continually evolving as organism targets are combined into multiplex assays, alternative specimen types are approved, and new or emerging STIs are identified. In contrast, the molecular methodologies have remained relatively unchanged since the mid1990's relying primarily upon signal amplification technologies including transcription mediated amplification (TMA) and polymerase chain reaction (PCR). Collectively, these commercial nucleic acid amplification test (NAAT) platforms have moved dichotomously in recent years towards high-throughput tests for high-volume screening, and point of care (POC) tests for more rapid turn-around time of results. Medium to high-throughput platforms serve hospital and reference laboratories that handle a high volume of test requests, whereas POC tests are geared more towards primary care and/or STD clinics.

Although high-throughput platforms are responsible for the vast majority of clinical screening and diagnosis of STIs worldwide, POC tests have the ability to provide rapid "while you wait" results thus facilitating immediate initiation of therapy if indicated. This paradigm is particularly useful in clinical settings where, after receiving test results from a reference or hospital laboratory, a follow up visit several days later is unlikely (e.g. urban STD clinics). POC tests also aid to reduce the common practice of syndromic management - the presumptive stat treatment of a group of signs/symptoms in the absence of STI test results and based solely on the most likely etiologic agent(s). Complications of this approach are discussed in the $M$. genitalium article in this FOCUS series whereby the current stat treatment guidelines for male nongonococcal urethritis (NGU) and non-gonococcal cervicitis may be enhancing the development of antibiotic resistance in this prevalent and emerging STI.

For decades, POC tests have been employed to aid in the accurate management of vaginitis caused by $T$. vaginalis and are discussed in the accompanying article in the series. It has been clear that $T$. vaginalis is a priority pathogen for recent commercial efforts in the field of STI diagnostics. We now appreciate the very large proportion of asymptomatic T. vaginalis infections in women, and it is expected that this organism will be an exceedingly common component of STI screening efforts as more FDA-approved tests are moved to market in the USA. Considering the widespread and subjective occurrences of vaginitis and related syndromes, it is also anticipated that requests for $T$. vaginalis testing will soon be as common or exceed those of CT/NG thereby further expanding the breadth and STI testing loads in diagnostic laboratories.

Unlike the other organisms discussed in this FOCUS series, $M$. genitalium is a sexually transmitted urogenital pathogen for which no FDA tests have been approved. In lieu of not having standardized tests for widespread use, clinical researchers have relied upon laboratorydeveloped tests (LDTs) to establish the basic epidemiology and disease spectra of $M$. genitalium infections in men and women. In this respect, the strides and enormous financial means necessary to establish and rationalize the need for a diagnostic test must be considered. For STIs, an inherent difficulty exists for linking pathogenic organisms with symptoms or specific sign of disease because a substantial proportion of subjects are asymptomatic. Up to $75 \%$ of women with $C$. trachomatis infections lack any symptoms and so no link between infection and clinical outcome can be made in a substantial number of those infected with the pathogen. As such, very large cohorts can be required to demonstrate a significant link between STIs and certain signs/symptoms of disease. Despite the required efforts and significant financial investment, the rationale for development and marketing of a FDA-approved $M$. genitalium NAAT is strong and discussed in this FOCUS series. Without widespread availability of an optimized and validated test, a substantial gap remains in our ability to informatively manage very common inflammatory urogenital syndromes for both men and women. A FDA-cleared test for $M$. genitalium would not only enhance the accuracy of patient treatment, but would also support the growing research community by facilitating clinical investigations of pathogenesis, epidemiology and optimized therapeutic strategies. These types of studies are absolutely imperative for expanding our understanding of $M$. genitalium as a urogenital pathogen.

In sharp contrast to $M$. genitalium, much is known about human papilloma virus (HPV) and its vaccinepreventable role in cervical cancer. $A$ vast and expanding market exists for HPV testing since NAATs 


\section{FOCUS: MOLECULAR DIAGNOSIS OF SEXUALLY TRANSMITTED INFECTIONS}

have been integrated into the 2012 cervical cancer management algorithms. HPV testing is unique among the STIs in that the test results are linked to the risk of developing high-grade cervical dysplasia, rather than simply the presence/absence of the target organism. Although HPV is by far the most common STI and etiology of cervical cancer, infections are transient in young women and detection of high-risk HPV types very rarely leads to cervical cancer. Therefore, HPV testing is only one part of a carefully designed series of algorithms that employ cytological screening (Papanicolaou or "Pap" smear) combined with downstream colposcopic and histologic examinations. For many years, cervical cancer screening has been recommended on an annual basis, but the recently updated guidelines have substantially increased the interval between screenings for most women. Adoption of these guidelines has extensive physiological and psychological ramifications for millions of American women now and in the coming years. In an accompanying article in this FOCUS series, we distill the current paradigm for cervical cancer screening in the USA, and address how HPV test results contribute to managing women with high-risk HPV infections and/or cervical dysplasia.

Together, this four article series is aimed to inform laboratory professionals, business thought leaders, medical/health educators, healthcare professionals, and the clinical diagnostics field at large about the past, current and future paradigms for STI management. For each pathogen, we have included a very brief summary and explanation of the clinical rationale that has led us to the current practices in the field. Medical laboratory professionals play an integral role in the health care system by providing diagnostic services that not only directly impact therapeutic management of patients, but also by offering their expertise in interpretation of the results in the exploding growth of MDx assays and menus of tests available to physicians. In that light, these professionals should have a basic grasp on the basis and rationale for a diagnostic test, and generally appreciate the downstream effects of reporting a positive/negative result. Finally, educators must become leaders in preparing clinically competent laboratory professionals by providing them with opportunities to expand their training and understanding of MDx. ${ }^{4}$ These concepts are discussed in the context of three important STIs with the goal of highlighting timely and pertinent affairs in the evolving field of STI diagnostics.

\section{REFERENCES}

1. World Health Organization. Prevalence and incidence of selected sexually transmitted infections, Chlamydia trachomatis, Neisseria gonorrhoeae, syphilis, and Trichomonas vaginalis: methods and results used by the WHO to generate 2005 estimates. Geneva, Switzerland: World Health Organization, 2011.

2. Satterwhite CL, Torrone E, Meites E, et al. Sexually transmitted infections among US women and men: prevalence and incidence estimates, 2008. Sex Transm Dis 2013;40(3): 187-93.

3. Owusu-Edusei Jr. K, Chesson HW, Gift TL, et al. The estimated direct medical cost of selected sexually transmitted infections in the United States, 2008. Sex Transm Dis 2013;40(3):197-201.

4. Rohde RE, Falleur DM, Kostroun P. Molecular Diagnostics CLS Course Design: Making it Real. Clin Lab Sci 2009;22(1):9-15. 